\title{
Femtosecond Laser Based Polarization Storage by Direct-Writing in Diazobenzene Copolymer Film
}

\author{
${\text { Yanlei } \mathrm{Hu}^{* 1} \text {, Zhoushun Zhang }}^{{ }^{*} 2}$, Yuhang Chen $^{{ }^{* 1}}$, Wenhao Huang ${ }^{{ }^{* 1}}$ and Qijin Zhang ${ }^{{ }^{2}}$ \\ ${ }^{*}$ Department of Precision Machinery and Precision Instrument, University of Science and Technol- \\ ogy of China, Hefei, Anhui 230026, P.R. China \\ E-mail:whuang@ustc.edu.cn \\ ${ }^{* 2}$ CAS Key Laboratory of Soft Matter Chemistry, Department of Polymer Science and Engineering, \\ University of Science and Technology of China, Key Laboratory of Optoelectronic Science and \\ Technology, Anhui Province, Hefei, Anhui 230026, P.R.China
}

\begin{abstract}
Femtosecond laser-based polarization storage by two-photon isomerization of chromophores has been intensively researched in recent years due to the potential capacity of multiple storage density. In this paper, we present a rewritable multilayer polarization storage method by direct-writing in a photoisomeric copolymer film. The influence of exposure time on the readout bit size is investigated. Dual-layer storage is achieved by placing a transparent spacer between two layers. It is found that the recorded information can be completely erased by circularly polarized laser. The contrast changes during erasing process are also studied.
\end{abstract}

DOI: $10.2961 /$ jlmn.2010.01.0014

Keywords: femtosecond laser, polarization storage, two-photon, isomerization, diazobenzene

\section{Introduction}

In order to fill the requirement of immense information memory, there are two different approaches to increase the optical storage capacity. Shortening the laser wavelength and increasing the numerical aperture (NA) of objective is one of the approaches to obtain smaller pit size, which we can conclude from the developing history of the compact disks (CD), digital versatile disks (DVD) and blue-ray disks (BD). However, the decrease of pit size encounters tremendous obstacle of the optically diffraction limit. The other approach is multi-dimensional storage, e.g. multilayer optical storage $[1,2]$, volumetric holographic technique [3] and wavelength multiplexed storage [4]. Multi-dimensional storage has been demonstrated to have the possibility to obtain a high storage density beyond terabits per cubic centimeter $\left(1\right.$ Tbits $\left./ \mathrm{cm}^{3}\right)$.

Two-photon absorption (TPA) is an unparalleled method for realizing multilayer data storage. It is wellknown that molecules can simultaneously absorb two photons under the effect of focused intense laser pulses, and the transition probability for TPA is proportional to the square of the intensity of the pulsed laser. With the help of the quadratic dependence, the chemical and physical processes can be confined to a highly localized area of volume $\lambda^{3}$ around the focal point, where $\lambda$ is the pulsed laser wavelength. A variety of materials have been used successfully in TPA 3D data storage, e.g. photochromic [5], photobleaching [6] and photorefractive materials [7].

Polymers containing azobenzene derivatives are considered as a kind of promising candidate for optical storage materials due to their erasable properties and low costs [8, 9]. Linearly polarized blue or green light can induce photoanisotropy by reorientation of the azobenzene group [10]. In detail, azobenzene derivatives have two geometric isomers, namely trans and cis. When illuminated by a specified linearly polarized light, trans-cis photochemical isomerization occurs by absorbing the light whose polarization direction is parallel to the molecules' dipole moment. The cis isomer then relaxes to a more stable trans state which axis is perpendicular to that of the initial state. The photoisomerization results in anisotropic refractive-index distribution, quantified by birefringence, which can be used in polarization multiplexed storage.

In this paper, we present a rewritable multilayer polarization storage method by direct-writing in a diazobenzene copolymer film. One-photon induced birefringence is discussed and two-photon induced birefringence is applied for polarization storage, which can increase the information capacity by orders of magnitude.

\section{Experimental}

The simplified model of the erasable polarization storage is illustrated in Fig.1. The molecules distribute randomly, resulting in optical isotropy in the initial sample. Under the illumination of linearly polarized light, the axes are oriented perpendicular to the polarization. Accordingly, the refractive index of the illuminated area becomes different from the untreated area. Circularly polarized light, which can be considered to have equal components in all directions, can randomize the molecular orientation. It suggests that circularly polarized light can be employed as erasing beam.

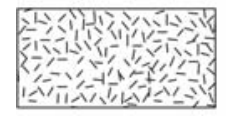

(a)

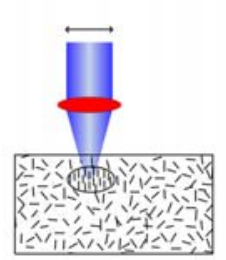

(b)

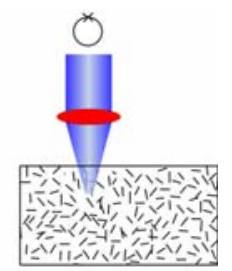

(c)
Fig.1. Simplified model of the erasable polarization storage. 
A diazobenzene copolymer, poly[(methyl meth acrylate)-co-4-\{(2-methacryloyloxyethyl)oxy $\}-4$ '-(4-

nitrophenylazo)azobenzene)] (poly(MMA-co-M2BAN)), as described in our previous work, was chosen as the storage medium [11]. We used a typical write-and-probe optical setup to measure the photoinduced birefringence. The sample was placed between two orthogonal polarizers. To probe the photoinduced birefringence, a linearly polarized $\mathrm{He}-\mathrm{Ne}$ laser with wavelength of $650 \mathrm{~nm}$ and power density of $30 \mathrm{~mW} / \mathrm{cm}^{2}$ was used as reading beam. The reading beam passed through the polarizer-sample-analyzer combination and then the transmission signal was detected. A linearly polarized $\mathrm{He}-\mathrm{Cd}$ laser $\left(441.6 \mathrm{~nm}, 300 \mathrm{~mW} / \mathrm{cm}^{2}\right)$ was used as writing beam to induce anisotropy. The polarization angle of the writing beam was set at $45^{\circ}$ with respect to the polarization of the reading beam. The experiment was performed at room temperature.

The experimental setup for recording, reading and erasing data was developed based on a confocal laser scanning microscope [12]. A femtosecond laser $(800 \mathrm{~nm}, 80 \mathrm{fs}, 80$ $\mathrm{MHz}$ ) is applied as the recording beam after being polarized and focused by an objective (40X, NA=0.65). White light generated by a halogen lamp was used as reading beam and a CCD was used to capture the magnified transmission images.

\section{Results and discussion}

Fig.2 shows one-photon writing-relaxation curve obtained in poly (MMA-co-B2MAN) film. Before the writing beam was switched on, no transmission of the probe beam was detected because of the molecular random orientation. After the writing beam was switched on, birefringence was induced and therefore transmission increased. In other words, photoinduced birefringence increased due to the molecular reorientation. The transmission reached saturation after about 20 minutes. It is because most of the molecules' dipole moments had been aligned perpendicular to the polarization of writing beam and no longer absorbed light. When the writing beam was turned off, the transmission decreased to a constant value, indicating a long-term and stable photoinduced birefringence. The induced birefringence $\Delta n$ can be obtained by the formula: $\Delta n=\frac{\lambda}{\pi d} \sin ^{-1} \sqrt{T}$, where $\lambda$ is the wavelength of monitoring beam, $d$ is the thickness of the sample, and $T$ is the transmittance [13].

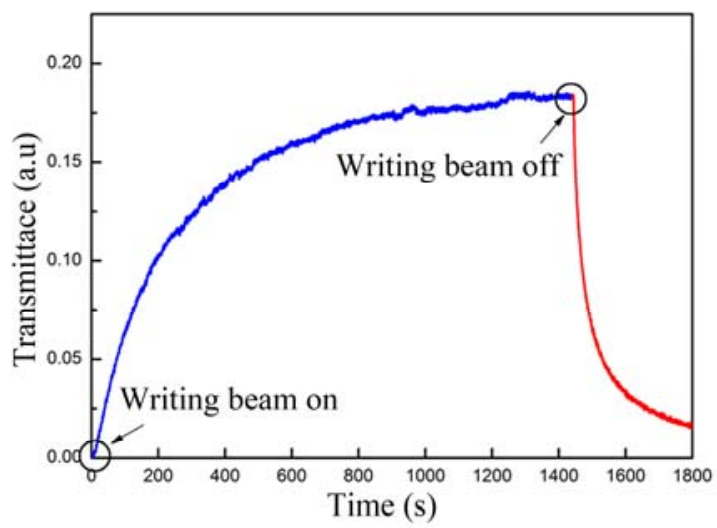

Fig.2. Writing and relaxation sequence obtained in poly (MMAco-B2MAN) film
Fig.3 shows the demonstration of two-photon induced polarization storage. The butterfly pattern was recorded by $0^{\circ}$ polarized laser beam and the other pattern, a floweret, was recorded by $45^{\circ}$ polarized laser beam. The average power of the recording laser is $16 \mathrm{~mW}$ and the exposure time for each bit is $35 \mathrm{~ms}$. The interval of adjacent bits is 4 $\mu \mathrm{m}$. The polarization of reading beam is (a) $0^{\circ}$, (b) $45^{\circ}$ and (c) $22.5^{\circ}$, respectively. It is demonstrated from Fig. 3(a) and Fig. 3(b) that two different kinds of information can be stored at the same region with negligible cross-talk. When the polarization of reading beam is set to $22.5^{\circ}$, the middle of the two recording beams, both the patterns appear and the intensity is lower as we expected. Fig. 4 shows the influence of exposure time on the recorded bit size. The bit size is estimated by the full-width at half-maximum (FWHM) of bit intensity. A nearly linear increase in bit size with exposure time is observed at the same excitation power of $16 \mathrm{~mW}$. It is also found that when the bits are recorded with exposure time below $15 \mathrm{~ms}$, the readout bits become indistinct and irregular. In order to achieve a proper balance between bit size and intensity, exposure time of $35 \mathrm{~ms}$ is chosen for recording.

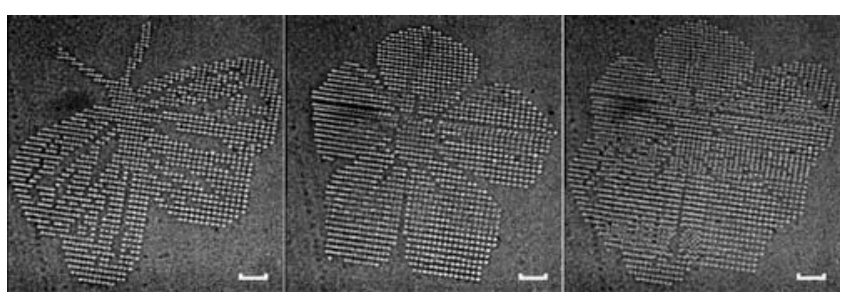

(a)

(b)

(c)

Fig.3. Demonstration of two-photon induced polarization storage. (a) and (b) are recorded by $0^{\circ}$ and $45^{\circ}$ polarized laser beam. The polarization of reading beam is (a) $0^{\circ}$, (b) $45^{\circ}$ and (c) $22.5^{\circ}$, respectively. The scale bar is $20 \mu \mathrm{m}$.

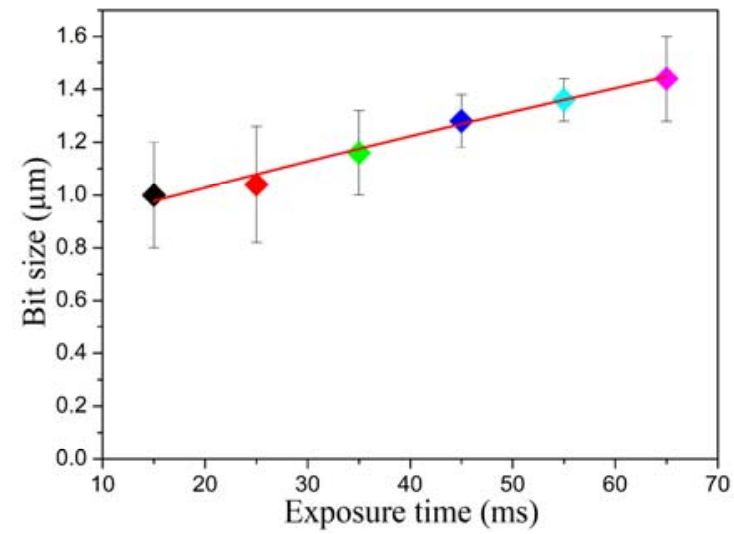

Fig.4. Influence of exposure time on the recorded bits size.

The demonstration of dual-layer storage is shown in Fig.5. A glass slide with thickness of about $100 \mu \mathrm{m}$ was placed between two layers as a transparent spacer. It is anticipated that $3 \mathrm{D}$ storage with more data layers and smaller separations can be achieved by sandwiching the copolymer sample between non-photosensitive films when preparing the recording medium. 


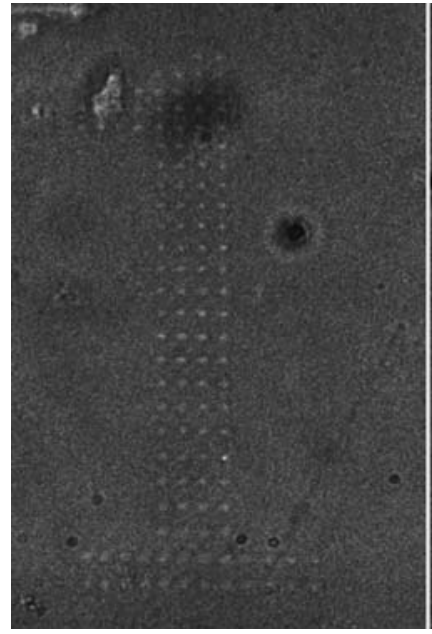

(a)

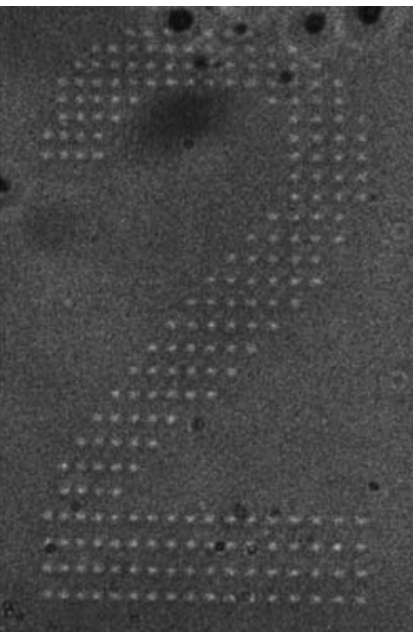

(b)
Fig.5. Demonstration of dual-layer storage. (a) the first layer; (b) the second layer. The separation between two layers is $\sim 100 \mu \mathrm{m}$.

The molecular reorientation can be relaxed by circularly polarized light. As shown in Fig.6, the letter "T" was firstly recorded in the medium and "E" was rewritten in the same region after " $T$ " was erased. The information was erased by using a tightly focused circularly polarized beam to scan the recorded area. The erasing power is $9 \mathrm{~mW}$ and the scanning speed is $75 \mu \mathrm{m} / \mathrm{s}$. The changes of contrast during erasing process are also studied (see Fig.7). Contrast is used to describe the difference between the maximum gray value (maximum signal intensity) and the minimum gray value (minimum signal intensity) in one image. In this paper, contrast is given by: $C=\log _{10}\left(I_{\max } / I_{\min }\right)$, where $C$ is the contrast, $I_{\max }$ and $I_{\min }$ are the maximum and minimum gray value respectively. Ideally, the contrast of the image read out before recording or after completely erased should be

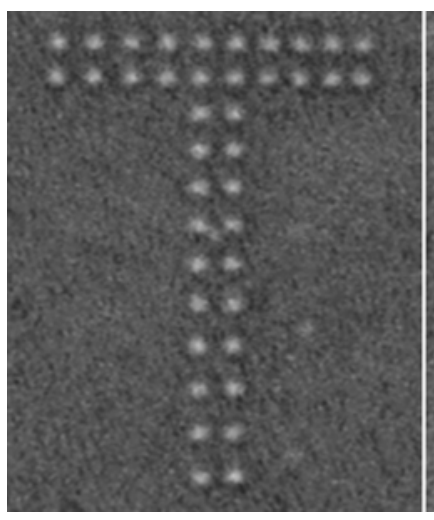

(a)

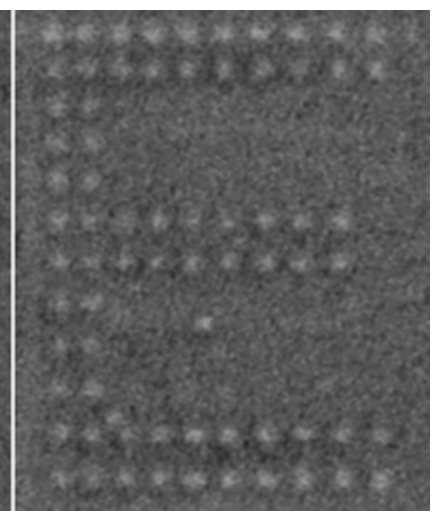

(b)
Fig.6. Demonstration of rewritable storage. (a) " $\mathrm{T}$ " is firstly recorded in the medium, and (b) "E" is rewritten in the same region after " $\mathrm{T}$ " is completely erased. zero. But because of the uniformity of light luminance, discontinuity of material in the sample and the thermal noise of CCD sensor, the gray value of the readout image is not always the same. The contrast of a "blank" image without recorded bits is around 0.188. It is found that the information can be completely erased after 3 cycles. The erasing speed can be improved with higher erasing power.

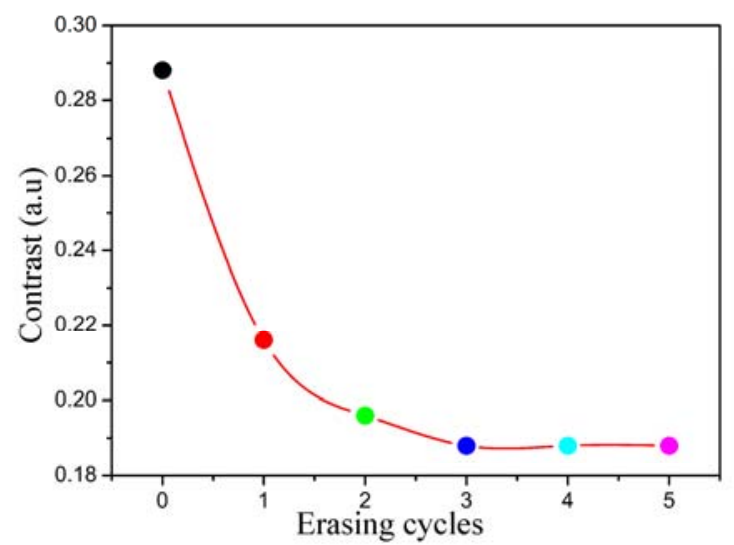

Fig.7. For an erasing circularly-polarized laser of power $9 \mathrm{~mW}$ and scanning speed of $75 \mu \mathrm{m} / \mathrm{s}$, bits contrast's dependence on the scanning cycles.

\section{Conclusion}

In conclusion, we have presented a rewritable multilayer polarization storage technique by femtosecond laser direct-writing in diazobenzene copolymer film, which has the potential to increase the storage density. The influence of exposure time on the readout bit size is investigated. The storage is also demonstrated to be erasable and rewritable, which is of great value in practical applications.

\section{Acknowledgements}

The work was supported by the National Natural Science Foundation of China (NSFC) 50875250.

\section{References}

[1] D. A. Parthenopoulos and P. M. Rentzepis, Science 245, (1989) 843

[2] D. Day and M. Gu, Appl. Opt. 37, (1998) 6299

[3] P. J. van Heerden, Appl. Opt. 2, (1963) 387

[4] P. Zijlstra, J. W. M. Chon and M. Gu, Nature 459, (2009) 410

[5] A. Toriumi, S. Kawata and M. Gu, Opt. Lett. 23, (1998) 1924

[6] D. Day and M. Gu, Appl. Opt. 37, (1998) 6299

[7] J. H. Strickler and W. W. Webb, Opt. Lett. 16, (1991) 1780

[8] M. Ishikawa, Y. Kawata, C. Egami, O. Sugihara, and N. Okamoto, Opt. Lett. 23, (1998) 1781

[9] X. Li, J. W. M. Chon, S. Wu, R. A. Evans, M. Gu, Opt. Lett. 32, (2007) 277

[10] S. Alasfar, M. Ishikawa, Y. Kawata, C. Egami,O. Sugihara, N. Okamoto, M. Tsuchimori, and O. Watanabe, Appl. Opt. 38, (1999) 6201

[11] Z. Zhang, Y. Hu, Y. Luo, Q. Zhang; W. Huang, G. Zou, Opt. Commun. 282, (2009) 3282 
[12] Y. Hu, Z. Zhang, Y. Chen, Q. Zhang, and W. Huang, Opt. Lett. 35, (2010) 46
[13] D.S. Correa, M.R. Cardoso, V.C. Goncalves, D.T. Balogh, L. De Boni, C.R. Mendonca, Polymer 49 (2008) 1562

(Received: July 9, 2009, Accepted: February 1, 2010) 